\title{
Downlink Scheduling and Rate Capping for LTE-Advanced Carrier Aggregation
}

\author{
Mieszko Chmiel, Jin Shi, David X. Zhou \\ LTE System Design \& Architecture, Nokia Siemens Networks, Beijing, China \\ Email: mieszko.chmiel@nsn.com,jin.shi@nsn.com, david.x.zhou@nsn.com
}

Received April, 2013

\begin{abstract}
Long Term Evolution (LTE) Carrier Aggregation (CA) was introduced by the Release-10 3GPP specifications. CA allows aggregation of up to 5 cells for a terminal; both downlink (DL) CA and uplink (UL) CA are supported by the 3GPP specifications. However, the first commercial deployments focus on the aggregation of two cells in the downlink. The benefits of LTE CA are increased terminal peak data rates, aggregation of fragmented spectrum and fast load balancing. In this paper, we analyze different strategies of DL scheduling for LTE CA including centralized, independent and distributed schedulers, we provide the corresponding simulation results considering UE data rate limitations and different traffic models. Also, we compare the performance of a single LTE carrier with LTE CA using the same total bandwidth.
\end{abstract}

Keywords: Long Term Evolution; Carrier Aggregation; Scheduling; Downlink; Bandwidth

\section{Introduction}

Carrier Aggregation is one of the Long Term Evolution Advanced features introduced by 3GPP in order to meet IMT-Advanced requirements of peak data rates of up to 1 Gbit/s in the DL and $500 \mathrm{Mbit} / \mathrm{s}$ in the UL [1-3]. In addition to the User Equipment (UE) peak data rate increase, another benefit of CA is the possibility for operators to aggregate fragmented spectrum. Also fast load balancing can be achieved with LTE-Advanced CA because of a UE with aggregated cells; the traffic can be scheduled on any of the aggregated cells on a Transmission Time Interval (TTI) basis.

The overview of LTE-Advanced CA is given in [4] and [5] while the CA impact on Radio Resource Management algorithms is presented in [6]. In [7], performance results with high number of DL aggregated cells are provided; furthermore, UL CA simulations results are reported in [8]. However, in this paper we focus on the aggregation of two DL cells since this is the first commercial deployment scenario for CA.

The performance of CA is highly dependent on the scheduling method used by the eNode B (eNB). The following three general scheduling principles can be used for CA.

- One centralized scheduler for all aggregated cells.

- Independent schedulers per aggregated cell $[9,10]$.

- Distributed and coordinated schedulers per cell [9, $10]$.
In this paper, we compare the above principles taking into account real-life effects such as traffic models and UE data rate limitations. Also, the performance comparison of DL CA with a single carrier of the same bandwidth (BW) is analyzed.

The paper is organized as follows. We discuss strategies for DL scheduling in section 2. Section 3 outlines simulation assumptions. In section 4 , we provide the simulation results. Finally, some conclusions are given in section 5 .

\section{CA Scheduling and Rate Capping Methods}

One centralized scheduler serving CA UEs and non-CA UEs of all aggregated cells can potentially offer the optimum performance. The frequency diversity over all aggregated cells can be exploited in scheduling of CA UEs. However, the challenge of this centralized scheduling method is the implementation complexity increased with the number of aggregated cells. In addition to lack of scalability, this method might be not feasible in future inter-eNB carrier aggregation scenarios.

Independent schedulers per aggregated cell represent the simple and scalable extension of single carrier scheduling. This option is expected to have worse performance compared to the centralized scheduling principle because the frequency diversity is exploited separately within each cell. Furthermore, the fairness between UEs 
can only be achieved and controlled on a cell basis; therefore, this solution is capable of neither achieving nor controlling throughput fairness between CA UEs and non-CA UEs. Also, it shall be noted that in fact the scheduling for CA cannot be fully independent per cell because there are UE data rate limits which shall not be exceeded when allocating resources on multiple cells to a CA UE. Such data rate limits are, for example, the 3GPP defined peak data rate of a given UE category [11] or the amount of UE data available for transmission in the buffer.

The distributed and coordinated schedulers per cell can achieve better performance for Carrier Aggregation compared to independent schedulers [10], the reason being that distributed schedulers can exploit frequency diversity over all aggregated cells in a similar way as the centralized scheduler. In this solution, each cell has its own scheduler; however, as opposed to the independent schedulers, the coordinated schedulers in aggregated cells communicate with each other for the purpose of optimizing scheduling metric calculation. In [9], it is shown that distributed and coordinated schedulers are optimal from the utility maximization point of view. This scheduling method can use the same or similar scheduling metric calculation as the centralized scheduling with the difference that the computation is distributed. The performance of distributed and coordinated schedulers for CA is on a par with centralized scheduling for full-buffer traffic and without considering UE data rate limits. However, if real-life effects like non-full-buffer traffic and finite UE data rate limits (e.g. the peak data rate) kick in, the performance of distributed and coordinated scheduling depends also on the rate capping method used to fulfill the CA UE data rate limits.

In this paper, we consider two methods for rate capping for CA UEs:

1) Static 50/50: the amount of data in the buffer and the peak data rate are divided equally to active serving cells.

2) Dynamic: the amount of data in the buffer and the peak data rate are divided to active serving cells proportionally to the UE throughput achieved on each of the active cells. Additionally, the division of data in the buffer might be adjusted if all data assigned to a given cell is drained in a TTI.

Another relevant topic is the performance comparison of distributed and coordinated CA scheduling with the performance of single-carrier scheduling in the same bandwidth. This comparison is impacted by higher protocol overhead of CA because separate Transport Blocks (TBs) are generated per each scheduled cell. On the other hand, a single cell of a bandwidth equal to the sum of the bandwidths of the aggregated cells will have a worse Channel State Information (CSI) and Resource Block Group (RBG) granularity.

\section{Simulation Assumptions}

A hexagonal regular cell layout in an urban deployment scenario with $500 \mathrm{~m}$ Inter-Site Distance (ISD) was simulated with frequency reuse 1 . The deployment area comprises 21 cells placed in a wrap-around model assuming a Typical Urban (TU) channel model. A pathloss model for small cells with PL slope of $37.6 \mathrm{~dB}$ per decade was used. Additional penetration loss of $20 \mathrm{~dB}$ for indoor coverage was taken into consideration [12]. Basic configuration parameters such as the pathloss model and antenna diagram were selected in accordance to [12].

The number of users within the simulation area was kept constant. Slow-moving subscribers were assumed. During the simulation run, a UE can change its serving cell by handover based on measurements (handover margin $3 \mathrm{~dB}$ ). The simulation model includes non-adaptive Hybrid Automatic Repeat Request (HARQ) with Chase Combining. The essential simulation parameters are listed in Table 1.

\section{Simulation Results}

\subsection{Carrier Aggregation and Single Cell without Physical Downlink Control Channel}

In this section, the performances of downlink intra-band $\mathrm{CA}$ and single-carrier operation are analyzed without Physical Downlink Control Channel (PDCCH) overhead. To evaluate the cell throughput performance, the same number of UEs (12) per cell scheduler will be set with full-buffer traffic. Other simulation parameters are listed in Table 2.

Table 1. Parameters of system simulation model.

\begin{tabular}{ll}
\hline \multicolumn{1}{c}{ Parameters } & \multicolumn{1}{c}{ Settings } \\
\hline Wrap around layout & 7 sites with 3 cells/site \\
Propagation scenario & Macro 1 (ISD 500 m) [12] \\
Carriers frequency 1 & Intra-band: 2 GHz \\
Carriers frequency 2 & Inter-band: $850 \mathrm{MHz}$ and 2 GHz \\
System bandwidth & CA: 2*10 MHz \\
Fast fading model & According to [13] \\
Indoor penetration loss & 20 dB (according to [12]) \\
Traffic model 1 & Full buffer [14] \\
Traffic model 2 & Constant Bit Rate \\
UE receiver & 2 RX (maximum ratio combining) \\
UE speed & 3 km/h \\
Scheduler & Proportional Fair \\
eNodeB power of cell & $40 \mathrm{~W}$ \\
Transmission mode & Closed loop MIMO, 2TX \\
CQI reporting mode & Mode-3 \\
Block Error Rate target & $10 \%$ \\
\hline
\end{tabular}


Figure 1 shows the average cell throughput normalized to $10 \mathrm{MHz}$ bandwidth.

CA of two $10 \mathrm{MHz}$ cells uses smaller Resource Block Group (RBG) and Channel Quality Indicator (CQI) granularity compared to a $20 \mathrm{MHz}$ cell. From the simulation result we see that the modified $20 \mathrm{MHz}$ simulation has $+3.54 \%$ higher cell throughput compared to normal 20 $\mathrm{MHz}$ simulation with worse granularity.

Considering the protocol overhead of additional transport blocks and no frequency diversity exploration across aggregated cells, the CA with independent scheduler has $-2.32 \%$ loss on cell throughput compared to a single $20 \mathrm{MHz}$ cell.

The CA with distributed scheduler is capable to have inter-scheduler communications. It recovers some of the frequency diversity gain from larger bandwidth. The distributed scheduler brings $+2.93 \%$ higher average cell throughput compared to independent schedulers.

From simulation without PDCCH, CA with distributed schedulers using two aggregated $10 \mathrm{MHz}$ cells can achieve performance similar to a single $20 \mathrm{MHz}$ cell.

Table 2. Settings for CA and single carrier simulations without PDCCH.

\begin{tabular}{lcccc}
\hline \multicolumn{1}{c}{ Parameters } & $\begin{array}{c}\text { Single } \\
20 \mathrm{MHz}\end{array}$ & $\begin{array}{c}\text { Modified } \\
\text { Single } \\
20 \mathrm{MHz}^{\mathrm{a}}\end{array}$ & $\begin{array}{c}\mathrm{CA} \\
\text { 2x10MHz } \\
\text { Option 1 }\end{array}$ & $\begin{array}{c}\text { CA } \\
\text { Op10MHz } \\
\text { Option 2 }\end{array}$ \\
\hline Number of cells & 21 & 21 & 42 & 42 \\
Number of UEs & 252 non-CA & 252 non-CA & $126 \mathrm{CA}$ & $126 \mathrm{CA}$ \\
per all cells & UEs & UEs & UEs & UEs \\
RBG size & 4 PRBs & 3 PRBs & 3 PRBs & 3 PRBs \\
CQI resolution & 4 PRBs & 3 PRBs & 3 PRBs & 3 PRBs \\
Scheduler & Centralized & Centralized & Independent & Distributed \\
PDCCH & Disabled & Disabled & Disabled & Disabled \\
\hline
\end{tabular}

${ }^{\mathrm{a}}$ The RBG and CQI resolution granularity is increased in simulation, but not possible by the 3GPP specification according to [15].

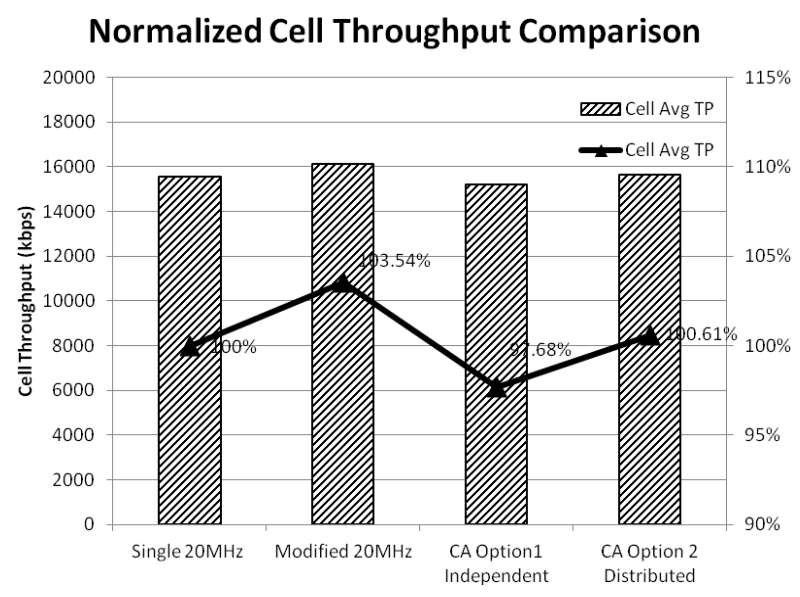

Figure 1. Cell throughput of CA and single carrier without PDCCH.

\subsection{Carrier Aggregation and Single Cell with Load-Adaptive PDCCH}

In this section, the performances of downlink intra-band CA and a single carrier are analyzed with the modeling of load-adaptive PDCCH. To focus on UE throughput, the same number of UEs (126) in simulation area will be set with the full buffer traffic model. Other simulation parameters are listed in Table 3.

Figure 2 shows the average and 5\%-ile of UE throughput.

With the PDCCH considered, the performance gap between CA and a single carrier becomes larger. There is $-6.98 \%$ loss on average UE throughput and $-11.39 \%$ on 5\%-ile UE throughput.

Figure 3 shows the utilization of PDCCH symbols and the utilization of Control Channel Elements (CCEs).

In CA, scheduling of the additional bandwidth requires additional PDCCH assignments. The higher number of orthogonal frequency-division multiplexing (OFDM) symbols for PDCCH reduces the number of OFDM symbols available for data transmission.

Table 3. Settings for CA and single carrier simulations with load adaptive PDCCH.

\begin{tabular}{lccc}
\hline \multicolumn{1}{c}{ Parameters } & $\begin{array}{c}\text { Single } \\
20 \mathrm{MHz}\end{array}$ & $\begin{array}{c}\text { Modified Single } \\
20 \mathrm{MHz}^{\mathrm{a}}\end{array}$ & $\begin{array}{c}\text { CA 2x10 MHz } \\
\text { Option } 2\end{array}$ \\
\hline $\begin{array}{l}\text { Number of cells } \\
\text { Number of UEs } \\
\text { per all cells }\end{array}$ & 21 & 21 & 42 \\
RBG size & 4 PRBs & 3 PRBs & 3 PRBs \\
CQI resolution & 4 PRBs & 3 PRBs & 3 PRBs \\
Scheduler & Centralized & Centralized & Distributed \\
PDCCH & Adaptive & Adaptive & Adaptive \\
\hline
\end{tabular}

${ }^{\mathrm{a}}$ The RBG and CQI resolution granularity is increased in simulation, but not possible by the 3GPP specification according to [15].

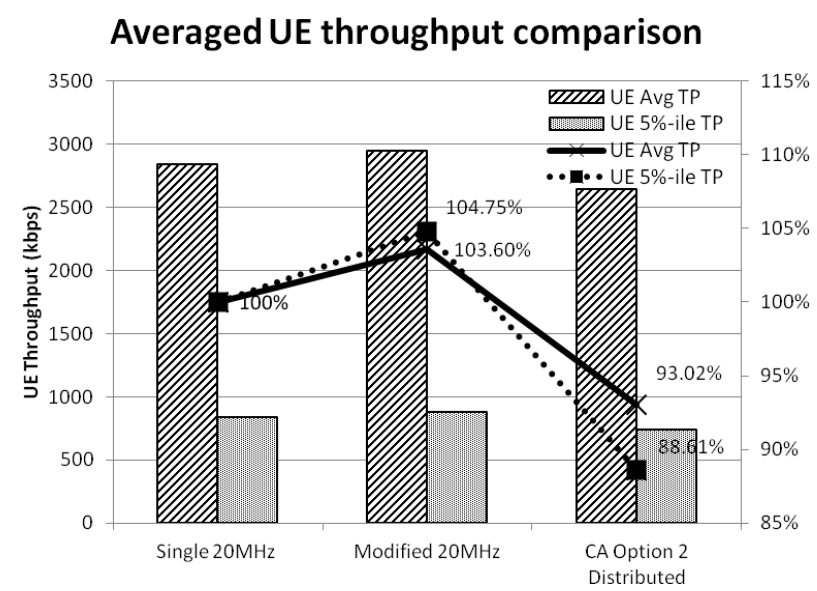

Figure 2. UE throughput of CA and single carrier with loadadaptive PDCCH. 


\subsection{Rate Capping on UE Buffer}

The problem of rate limitation of CA UEs is described in section 2. There are several solutions to rate capping due to the UE buffer, which are investigated in this section; rate capping due to the peak data rate is investigated in section 4.4. Table 4 lists the solutions to rate capping due to the UE RLC buffer which are compared in our simulation

The simulation assumption for RLC buffer rate capping simulations can be found in Table 1 with the second traffic model - Constant Bit Rate (CBR) and inter-band CA. Each user has a $1 \mathrm{Mb} / \mathrm{s}$ CBR service. The simulation results are shown in Figure 4.

The simulation results are analyzed in terms of number of allocated Physical Resource Blocks (PRBs) per cell for the CBR service. From the results it can be seen that the ideal mode is the most efficient method for each number users. The dynamic mode is superior to the static mode with 50\%-50\% split.

\subsection{Rate Capping on Peak Data Rate}

Table 5 shows the solutions to rate capping due to the UE peak data rate which are compared in our simulation.

The peak data rate of the UE in our simulations is 51.024 Mbps which is based on UE category 2 according to [11].

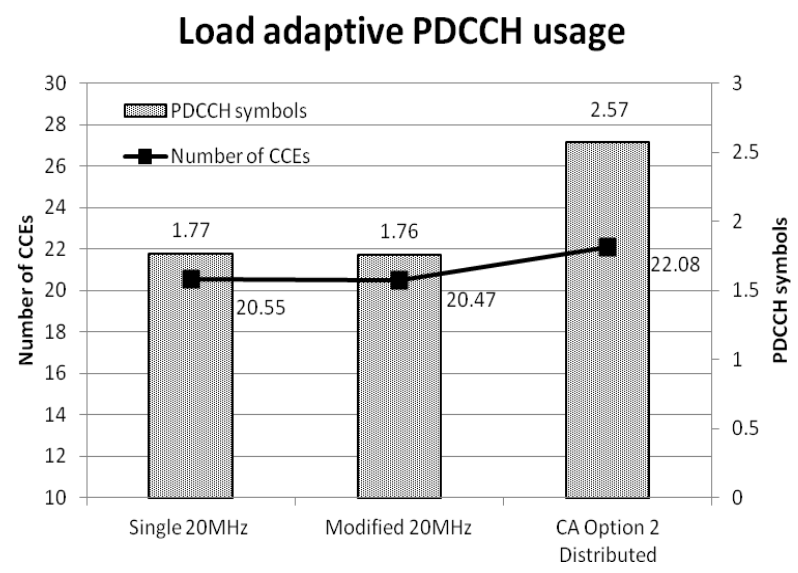

Figure 3. PDCCH symbols of CA and single carrier.

Table 4. Solutions to RLC buffer rate capping.

\begin{tabular}{cl}
\hline Solution & \multicolumn{1}{c}{ Description } \\
\hline Ideal & Close to genie-aided \\
Static & $\begin{array}{l}50 \% \text { of the RLC buffer allocated to the PCell and } \\
\text { remaining to the SCell statically }\end{array}$ \\
Dynamic & $\begin{array}{l}\text { X\% RLC buffer allocated for PCell and }(100-\mathrm{X}) \% \\
\text { for SCell dynamically }\end{array}$ \\
\hline
\end{tabular}

The simulation assumption for rate capping due to the UE peak data rate can be seen in Table 1 with the first traffic model - Full Buffer and inter-band CA. Figure 5 shows the simulation results.

The simulation results are analyzed in terms of average user throughput for the full-buffer service. From the results it can be seen that the difference between different modes in higher number of users is very small. However, in case of a very low number of users such as 1 or 2, the ideal mode is superior to other modes while the dynamic mode is slightly better than the static mode with $50 \%$ $50 \%$ split.

\section{Num of Allocated PRB Per Cell}

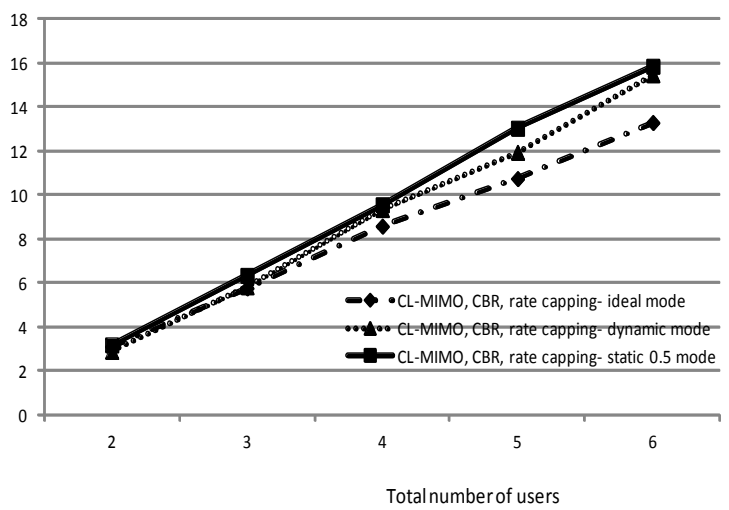

Figure 4. Simulation results of RLC buffer rate caping.

Table 5. Solutions to peak data rate capping.

\begin{tabular}{cl}
\hline Solution & \multicolumn{1}{c}{ Description } \\
\hline Ideal & Close to genie-aided \\
Static & $\begin{array}{l}\text { 50\% of UE Peak Data Rate allocated to the PCell and } \\
\text { remaining to the SCell statically }\end{array}$ \\
Dynamic & $\begin{array}{l}\mathrm{X} \% \text { UE Peak Data Rate allocated for PCell and } \\
(100-\mathrm{X}) \% \text { for SCell dynamically }\end{array}$ \\
\hline
\end{tabular}

Average User Througphut (kbps)

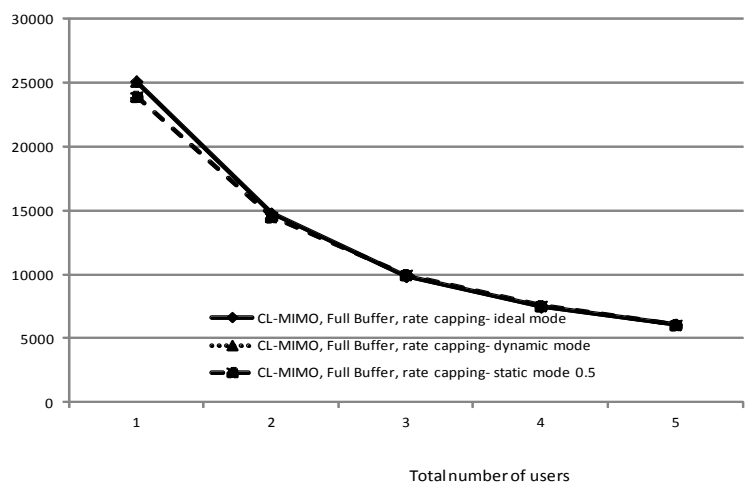

Figure 5. Simulation Results of Rate Capping on Peak Data Rate. 


\section{Conclusions}

In this paper, we discussed and simulated rate capping methods for LTE-A DL Carrier Aggregation scheduling. Such rate capping is required due to non-full-buffer traffic and other practical UE data rate limits when independent or distributed/coordinated scheduling is used for CA. Also, we provided simulation results comparing the performance of CA scheduling methods with a single cell.

Based on the analysis and the simulations in this paper, we draw the following conclusions. Aggregated cells can have spectral efficiency similar to a single cell of the same bandwidth assuming the same number of full- buffer UEs per cell. Distributed and coordinated schedulers provide better performance for DL CA compared to independent schedulers. The analyzed rate capping solutions provide good performance; the dynamic solution outperforms the static solution.

\section{Acknowledgements}

The authors of this paper would like to thank Hans Kroener and Harald Steinhaus for their valuable comments and help.

\section{REFERENCES}

[1] S. Parkvall, E. Dahlman, A. Furuskar, A. Kangas, M. Olsson, S. Wanstedt and K. Zangi, "LTE-Advanced Evolving LTE towards IMT-Advanced,” IEEE 68th Vehicular Technology Conference VTC 2008-Fall, 21-24 September 2008, pp. 1-5. 10.1109/VETECF.2008.313

[2] S. Parkvall, A. Furuskar and E. Dahlman, "Evolution of LTE toward IMT-advanced," IEEE Communications Magazine, Vol. 49, February 2011, pp. 84-91. doi:10.1109/MCOM.2011.5706315

[3] S. Parkvall, E. Englund, A. Furuskar, E. Dahlman, T. Jönsson and A. Paravati, "LTE Evolution towards IMTadvanced and Commercial Network Performance,” IEEE International Conference on Communication Systems (ICCS), 17-19 November 2010, pp. 151-155. doi:10.1109/ICCS.2010.5686370

[4] R Ratasuk, D. Tolli and A. Ghosh, "Carrier Aggregation in LTE-Advanced," IEEE 71st Vehicular Technology Conference (VTC 2010-Spring), 16-19 May 2010, pp. 1-5. doi:10.1109/VETECS.2010.5493902
[5] Z. K. Shen; A. Papasakellariou, J. Montojo, D. Gerstenberger and F. L. Xu, "Overview of 3GPP LTE-advanced Carrier Aggregation for 4G Wireless Communications," IEEE Communications Magazine, Vol. 50, Feruary 2012, pp. 122-130. doi:10.1109/MCOM.2012.6146491

[6] K. I. Pedersen, F. Frederiksen, C. Rosa, H. Nguyen, L. G. U. Garcia and Y. Y. Wang, "Carrier Aggregation for LTE-advanced: Functionality and Performance Aspects," IEEE Communications Magazine, Vol. 49, 2011, pp. 89-95. doi:10.1109/MCOM.2011.5783991

[7] I. Shayea, M. Ismail and R. Nordin, "Capacity Evaluation of Carrier Aggregation Techniques in LTE-Advanced system," International Conference on Computer and Communication Engineering (ICCCE), 2012, pp. 99-103. doi:10.1109/ICCCE.2012.6271160

[8] H. Wang, C. Rosa and K. Pedersen, "Performance of Uplink Carrier Aggregation in LTE-Advanced Systems,” IEEE 72nd Vehicular Technology Conference Fall (VTC 2010-Fall), 6-9 September 2010, pp. 1-5. doi:10.1109/VETECF.2010.5594543

[9] Y. Y. Wang, K. I. Pedersen, T. B. Sorensen, P. E. Mogensen, "Utility Maximization in LTE-Advanced Systems with Carrier Aggregation,” IEEE 73rd Vehicular Technology Conference (VTC Spring), 15-18 May 2011, pp. 1-5. doi:10.1109/VETECS.2011.5956494

[10] Y. Y. Wang, K. I. Pedersen, T. B. Sorensen and P. E. Mogensen, "Carrier Load Balancing and Packet Scheduling for Multi-carrier Systems," IEEE Transactions on Wireless Communications, Vol. 9, May 2010, pp. 1780-1789. doi:10.1109/10.1109/TWC.2010.05.091310

[11] 3GPP TS 36.306 v10.9.0 "Evolved Universal Terrestrial Radio Access (E-UTRA); User Equipment (UE) radio access capabilities (Release 10),” TSG RAN.

[12] 3GPP TR 25.814 v7.1.0, "Physical Layer Aspects for Evolved Universal Terrestrial Radio Access (UTRA) (Release 7),” TSG RAN.

[13] ETSI TR 101112 v3.2.0, "Procedures for the Choice of Radio Transmission Technologies of the UMTS (UMTS 30.03 version 3.2.0)," SMG.

[14] 3GPP R1-070674, "LTE Physical Layer Framework for Performance Verification,” Orange, China Mobile, KPN, NTT DoCoMo, Sprint, T-Mobile, Vodafone, Telecom Italia, 3GPP TSG RAN1\#48.

[15] 3GPP TS 36.213 v10.9.0 "Evolved Universal Terrestrial Radio Access (E-UTRA); Physical layer procedures (Release 10),” TSG RAN. 